\title{
INVESTIGAÇÃO DE GRUPOS ESTRATÉGICOS \\ NA INDÚSTRIA DE LATICÍNIOS POR MEIO DA \\ ABORDAGEM MULTIVARIADA
}

\section{INVESTIGATION OF STRATEGIC GROUPS IN THE INDUSTRY OF DAIRY PRODUCTS BY MULTIVARIATE APPROACH}

\author{
MARCO AURÉLIO MARQUES FERREIRA \\ Doutor em Economia Aplicada pela Universidade Federal de Viçosa (UFV). \\ Professor adjunto da Universidade Federal de Viçosa (UFV). \\ Departamento de Administração, Campus UFV - Viçosa - MG - CEP 36570-000 \\ E-mail: marcoaurelio@ufv.br
}

\section{LUIZ ANTÔNIO ABRANTES}

Doutor em Administração pela Universidade Federal de Lavras (UFLA).

Professor adjunto da Universidade Federal de Viçosa (UFV).

Departamento de Administração, Campus UFV - Viçosa - MG - CEP 36570-000

E-mail: abrantes@ufv.br

RONALDO PEREZ

Doutor em Engenharia de Alimentos pela Universidade Estadual de Campinas (UNICAMP).

Professor adjunto da Universidade Federal de Viçosa (UFV).

Departamento de Tecnologia de Alimentos, Campus UFV - Viçosa - MG - CEP 36570-000

E-mail:rperez@ufv.br 


\section{RESUMO}

Este trabalho teve como objetivo analisar os agrupamentos estratégicos na indústria de laticínios. O estudo fundamentou-se na abordagem de grupos estratégicos e teve como modelo analítico a abordagem multivariada de dados. A análise fatorial foi utilizada para a construção dos componentes estratégicos, e empregou-se a análise de cluster para definir os grupos estratégicos. Participaram da pesquisa I88 empresas de laticínios do Estado de Minas Gerais. Os resultados ressaltaram a presença de fatores estratégicos na indústria e possibilitaram construir três grupos estratégicos, sendo eles: pequeno porte especializado, alto porte diversificado e médio porte diferenciado.

\section{PALAVRAS-CHAVE}

Grupos estratégicos; Análise multivariada; Agrupamento; Análise fatorial; Indústria de laticínios.

\section{ABSTRACT}

The objective of this paper was to analyze the strategic group in the industry of dairy products. The study was based in the approach of strategic groups and it used as methodology the multivariate analysis of data. The Factorial Analysis was used for the construction of the strategic components and the cluster analysis was used to define the strategic groups. They participated in the research I 88 companies of dairy products of the state of Minas Gerais. The results pointed out the presence of strategic factors in the industry and they made possible to build three strategic groups: Small and Specialized companies; High and Diversified companies and Medium and Differentiated companies.

\section{KEYWORDS}

Strategic groups; Multivariate analysis; Cluster; Factorial analysis; Dairy products. 


\section{INTRODUÇ ̃̃O}

A produção de leite no Brasil destaca-se como uma das principais atividades agropecuárias em razão de sua capacidade de geração de emprego e renda e conexão com outros setores agroindustriais. A sua importância socioeconômica pode ser constatada pela posição que ocupa no agronegócio brasileiro, estando entre os principais setores em geração de renda nacional e arrecadação tributária. De 2004 para 2005, o setor passou da sexta para a quinta posição no ranking de valor bruto da produção agropecuária nacional, apresentando crescimento de $5,6 \%$, fato que promoveu o seu equilíbrio diante da queda de alguns segmentos ocorrida no mesmo período.

QUADRO I

VALOR BRUTO DA PRODUÇÃO AGROPECUARIA BRASILEIRA EM 2004 E 2005

\begin{tabular}{lccc}
\hline \multirow{2}{*}{ PRODUTOS } & \multicolumn{1}{c}{ VALOR BRUTO DA PRODUÇÃO (R\$ MILHÕES) } & VARIAÇÃO \\
\cline { 2 - 3 } & 2004 & 2005 & $(2004 / 2005)$ \\
\hline Carne bovina & 32.208 & 30.628 & $-0,049$ \\
\hline Soja & 36.729 & 25.196 & $-0,314$ \\
\hline Frango & 16.403 & 16.533 & 0,008 \\
\cline { 1 - 2 } Cana-de-açúcar & 12.525 & 13.402 & 0,070 \\
\hline Leite & 11.900 & 12.572 & 0,056 \\
\hline Milho & 13.805 & 10.240 & $-0,258$ \\
\hline Café beneficiado & 8.813 & 9.572 & 0,086 \\
\hline Suíno & 6.392 & 6.802 & 0,064 \\
\hline Arroz & 8.847 & 6.620 & $-0,252$ \\
\hline Laranja & 2.991 & 3.135 & 0,048 \\
\hline
\end{tabular}

Fonte: Embrapa Gado de Leite (2006).

Entre os parques industriais de produção e processamento nacional, a Região Sudeste destaca-se pela concentração da produção que atinge aproximadamente $39,4 \%$ do total nacional, pelos maiores centros de consumo e por maior parte das indústrias de laticínios do país. 
Nessa região, o Estado de Minas Gerais exerce liderança no agronegócio de lácteos, com expressiva produção, ocupando o primeiro lugar na produção nacional com $28, \mathrm{I} \%$ do total produzido e apresentando crescimento de $83, \mathrm{I} 4 \%$, de I9 85 a 2005 .

Apesar desse cenário favorável, o Estado é caracterizado pela heterogeneidade das diferentes unidades produtivas de lácteos. De um lado, observa-se a presença de grande número de organizações de pequeno porte altamente especializadas; de outro, estão as grandes organizações processadoras com intensa agregação de valor e diversificação de produtos. Em uma vertente mais regionalizada, observam-se também as organizações do tipo "nicho" discorridas por Ferreira (2005). Essas organizações têm como foco estratégico o posicionamento defensivo no mercado, atrelando agregação de valor à escala moderada, com o propósito de proteger-se da intensa rivalidade presente no segmento de processamento em alta escala.

Em razão da presença desses diferentes portes em uma mesma indústria, torna-se lícito supor que boa parte das mudanças que tem afetado a organização industrial do setor lácteo está sendo determinada pelos custos de produção, pela capacidade de investimento da organização, pelo poder de penetração das marcas, pela variedade da cesta de produtos e pelas parcerias e alianças estratégicas associadas a economias de escala e ganhos de eficiência apontados em Ferrier e Porter (I99I), Melo (2003) e Ferreira e Braga (2003, 2004).

Tal processo tem estimulado as unidades produtivas da indústria de laticínios a introduzirem mecanismos de ajustamento estratégicos, tais como posicionamento em relação à diversificação, ao investimento profissional e à realização de parcerias para a ampliação da escala de processamento ou distribuição.

Na literatura econômica, existem diversos trabalhos que promovem associações entre o tipo de organização e o seu desempenho na indústria de laticínios. Entre os trabalhos que têm suportado a existência de diferentes agrupamentos na indústria de laticínios, destacam-se Van Bekkum (200I) e Ferreira (2005).

Outros trabalhos tomam como referência para análise do desempenho o comportamento estratégico da organização. Nessa vertente, Lannes (2002) atribuiu melhor desempenho às maiores cooperativas de lácteos, bem como àquelas que promovem maior diferenciação dos seus produtos. Penrose (I959), na explanação sobre a teoria do crescimento da firma, dissertou sobre a relação entre eficiência e tamanho da organização, afirmando que as maiores firmas têm maior possibilidade de obter melhor desempenho. Para essa autora, essa influência se verifica em razão de as maiores empresas deterem maiores recursos produtivos e acesso aos processos tecnológicos mais complexos e parcimoniosos do que as pequenas empresas. Porter (I986), por sua vez, associou o desempenho das organizações a seu posicionamento estratégico mercadológico, afirmando que o 
propósito de uma unidade empresarial é encontrar uma posição no mercado em que se possa defender contra as forças que atuam sobre ela ou influenciá-las em seu favor. Assim, ações estratégicas que determinem seu posicionamento são variáveis importantes na explicação da eficiência.

Outro comportamento estratégico apresentado em manuais de administração e economia, como promotor do desempenho, tem sido a concentração em torno de seu negócio-chave e a conseqüente produção em escala. Essa postura é fundamentada, sobretudo, na teoria da firma, segundo a qual a concentração, somada ao máximo aproveitamento da força de trabalho e dos fatores tecnológicos, permite a redução do custo unitário, pelas chamadas economias de escala, sendo também forte argumento contra a diversificação.

Sob outra ótica, a diferenciação também é vista como promotora da eficiência pelo fato de a agregação de valor ao produto permitir melhor resultado na relação produto/insumo e nas benesses associadas à fidelidade do consumidor às marcas. Esse fator poderia estar associado também ao fato de os produtos mais elaborados terem maior elasticidade de renda, o que permite às unidades produtivas que trabalham com intensa agregação de valor ao produto aproveitar-se das benesses associadas aos períodos de estabilidade e aumento do poder aquisitivo da classe consumidora.

Disso decorre que o agrupamento estratégico das organizações na indústria de laticínios é, por demais, explorado em estudos científicos, embora não se tenha a real consciência da existência de fatores estratégicos capazes de possibilitar tal classificação, o que ressalta o lapso teórico-científico nessa área.

Como não existe, ainda, consenso sobre a existência de fatores condicionantes capazes de direcionar as opões estratégicas das organizações, muito se tem questionado sobre a possibilidade de criar agrupamentos estratégicos nesse setor como forma de introduzir mecanismos e políticas públicas de incentivo à eficiência e competitividade no setor. Nessa direção, o presente trabalho se propõe verificar a existência de elementos condicionantes da organização estratégica nas unidades processadoras de lácteos, visando construir diferentes agrupamentos estratégicos para o setor.

\section{REFERENCIAL TEÓRICO}

\subsection{CLASSIFICAÇÃO ORGANIZACIONAL}

As classificações são resultantes de um processo de agrupamento decorrente de alguma metodologia empírica ou estatística específica. As classificações mais comuns são aquelas decorrentes de processos estatísticos de agrupamento, dado 
o caráter de confiança atribuído a essa metodologia, amparada por vasta literatura. Nessa ótica, uma classificação decorrente de processo de agrupamento deve ser vista como um sistema tipológico comprometido com certos componentes que minimizem a subjetividade do pesquisador, atendendo a requisitos teóricos sustentatórios.

De acordo com Schendel e Hofer (I979), toda classificação está pautada em algum atributo que permita descrever as características fundamentais das unidades produtivas no que diz respeito às suas competências, aos seus recursos e às suas oportunidades e ameaças, visando à sua adequação ao ambiente organizacional.

Para Chrisman et al. (1988), uma classificação deve atender aos atributos de diferenciação, identificação, generalização e recuperação da informação.

A diferenciação pode ser percebida como o caráter de distinção entre cada um dos agrupamentos, enquanto generalização diz respeito à possibilidade de realização de estudos e inferências para todas as unidades do agrupamento. A identificação está associada ao fato de se poderem realizar inferências pontuais sobre as unidades produtivas singulares de um agrupamento, sem perder a consistência com o grupo. Por fim, é necessário que um sistema de classificação não apenas acumule informações, mas permita também seu armazenamento e sua recuperação para fins de análise (CHRISMAN et al., I988).

Existem diversos estudos e ensaios literários sobre a construção de agrupamentos estratégicos e classificação de cooperativas, alguns dos quais discorridos em Murray (1983), Barton (1989), Cook (I995), Van Dijk (1996), Cook e Tong (I997) e Nilsson (I999).

De acordo com Van Bekkum (200I), a grande limitação entre essas classificações de cooperativas é que elas não aplicam critérios explícitos e uniformes pelos quais se possam agrupar as organizações de forma coerente. Nesse sentido, Kyriakopoulos (2000) destaca que os modelos de agrupamento propostos têm a propriedade de sobrepor características cooperativas com características de mercado, incorrendo em falhas decorrentes do fato de esses modelos serem associados mais a critérios vagos de classificação do que a conceitos mensuráveis, que permitam identificar e descrever as relações distintivas entre as variáveis que compõem cada agrupamento.

Alguns trabalhos têm procurado definir critérios mais objetivos de classificação, entre os quais se destacam os de Hendrikse e Veerman (I997), que esboçaram uma tipologia de cooperativas com base em atributos de distinção, como estrutura financeira, estrutura de governança, perfil dos contratos de aquisição de matéria-prima e regulamentos de entrada e saída da sociedade. Nesse ponto, os autores salientaram que distintas cooperativas podem ter os mesmos atributos, porém existem diferenças consideráveis em suas magnitudes, bem como na direção estratégica adotada, o que determina a sua classificação. 
Outros trabalhos têm esboçado agrupamentos de cooperativas com base nas estratégias genéricas de Porter (I986), em razão, principalmente, de esse modelo assinalar três vetores estratégicos característicos da órbita cooperativa: liderançacusto, diferenciação e nicho.

Com base nos conceitos expostos, Van Bekkum (200I) e Ferreira (2005) foram os estudiosos que, mais recentemente, esboçaram classificações lastreadas em critérios objetivos. Van Bekkum (200I), em artigo seminal, apresentou classificação para as cooperativas de lácteos, baseando-se em três fatores estratégicos: a) diferenciação, associada ao interesse e à capacidade de a cooperativa ostentar uma marca; b) estrutura, refletindo maior ou menor vocação da cooperativa à coletividade; e c) estratégia de custo, refletida na escala de produção. Assim, em razão do cruzamento dos vetores de diferenciação, nicho e valor adicionado, o referido autor classificou as cooperativas em quatro agrupamentos sustentáveis: cooperativas de nicho, regionais, de commodities e de valor adicionado.

Alguns outros exemplos da implementação dessa análise podem ser absorvidos dos trabalhos de Herbert e Deresky (I987), Hergert (I987), Wright et al. (I99I) e Matias (I992).

\subsection{ABORDAGEM DOS AGRUPAMENTOS ESTRATÉGICOS}

De acordo com Athanassopoulos (I995), agrupamentos ou grupos estratégicos podem ser definidos como um conjunto de empresas que atuam dentro do mesmo ambiente industrial, seguem estratégias semelhantes e exibem características simétricas em várias dimensões, a exemplo de estrutura de custo, grau de diferenciação de produto, posicionamento mercadológico, desempenho e comprometimento de recursos. Nessa ótica, grupos estratégicos podem ser identificados e classificados em todos os setores em que participa um número significativo de organizações, a exemplo da indústria de laticínios.

A teoria de grupos estratégicos tem origem nos estudos das semelhanças de comportamentos estratégicos das organizações, desenvolvendo-se, exponencialmente, nas duas últimas décadas, com aplicações em diversos setores industriais, a exemplo dos setores farmacêutico (BOGNER et al., I996, I998; COOL; SCHENDEL, I988), de supermercado (LEWIS; THOMAS, I990, I994), seguros (FIEGENBAUM et al., I993; FIEGENBAUM; THOMAS, I994; BOGNER et al., I996), bebidas (DAY et al., I995), microcomputadores (LAWLESS; ANDERSON, I996) e tecnologia de informação (DUYSTERS; HAGEDOORN, I995), entre outros.

De acordo com essa teoria, há diferentes justificativas para realizar o agrupamento das empresas em razão de suas semelhanças. Caves e Porter (I977) e Porter (I986) constataram que o advento dos grupos estratégicos está condicio- 
nado ao fato de, em uma mesma indústria, as empresas reagirem de formas diferentes no que diz respeito às suas estratégias competitivas. Nessa ótica, Hatten e Schnedel (I977) e Hatten et al. (I978) destacaram a importância de visualizar a possibilidade de agrupar esses comportamentos das empresas, gerando a idéia de grupos estratégicos, em vez de decompor a indústria em grupos de empresas, por meio de estratificações generalistas ou indiscriminadas.

Para Barney (I99I), Mahoney e Pandian (I992) e Reger e Huff (I993), os grupos de empresas originam-se de organizações que mantêm fortes semelhanças em ativos, estrutura ou desempenho, o que as leva a um conjunto de comportamentos semelhantes, que permite distingui-las em relação a outro agrupamento.

Assim, a análise dos grupos estratégicos é, via de regra, precedida da composição dos grupos, o que é realizado com base nas similaridades e diferenças entre as organizações.

Nesse sentido, os benefícios de uma análise condicionada pela composição de grupos estratégicos estão ligados à exploração das características físicas e comportamentais das organizações que compõem um mesmo escopo estratégico, visando interpretar a razão de suas diferenças em dado atributo. Esse atributo pode ser desempenho financeiro, crescimento operacional, parcela de mercado ou eficiência, a exemplo deste trabalho.

A maior crítica à abordagem de grupos estratégicos está na orientação empírica e nos atributos discricionários que compõem a escolha das variáveis estrategicamente pertinentes à construção da análise de agrupamento (BARNEY; HOSKISSON, I990).

Medidas de semelhanças estratégicas entre empresas são objeto de estudo de diversos trabalhos, a exemplo dos de Barney e Hoskisson (I990), Ketchen e Shook (1993), Thomas e Carroll (i994) e Duysters e Hagedoorn (I995). De acordo com Barney e Hoskisson (I990) e Ketchen e Shook (I993), medidas de semelhanças podem ser criadas via processo dedutivo ou intuitivo, o que dependerá do rigor metodológico da pesquisa.

Nos últimos anos, os trabalhos têm enfatizado duas linhas, abordando a participação de cada organização no grupo estratégico ou o comportamento dos agrupamentos em si. Assim, a vasta literatura sobre avaliação de grupos estratégicos tem sido complementada por trabalho de pesquisa que busca explorar a convergência de métodos alternativos de formação dos grupos estratégicos, via critérios que enfatizam a contribuição dos elementos que compõem o grupo (NATH; GRUCA, I997; PETERAF; SHANLEY, I997).

De acordo com Athanassopoulos (I995), a crescente utilização da análise de grupos estratégicos chamou atenção para o uso de métodos quantitativos apropriados à composição dessa abordagem. Essas metodologias incluem uso de teoria dos jogos (KUMAR et al., I990), análise multivariada (DUYSTERS; 
HAGEDOORN, I995), otimização (LAWLESS; ANDERSON, I996), regressão linear (GORDON; MILNE, I999) e construção de fronteiras de eficiência (SCHEFCZYK, I993; FIEGENBAUM; THOMAS, I994; DAY et al., I995; ATHANASSOPOULOS; BALLANTINE, I995).

\section{METODOLOGIA}

\section{1 ÁREA DO ESTUdO E FONTE DOS DADOS}

As unidades produtivas que compõem a amostra são formadas por I88 laticínios localizados na mesorregião do sul e sudoeste de Minas Gerais, cujos dirigentes foram entrevistados, com a aplicação de questionário semi-estruturado, no período entre o primeiro e o segundo semestre de 2005. Essas organizações representam, aproximadamente, $72 \%$ de todas as unidades produtivas de lácteos da região.

A escolha dessa mesorregião se justifica por sua expressiva participação na atividade leiteira de Minas Gerais, respondendo por, aproximadamente, 26\% do número de laticínios do Estado. Nesse ponto, ressalta-se, também, que esse Estado é pioneiro na produção e no processamento de lácteos no país.

\subsection{ANÁLISE EXPLORATÓRIA DOS DADOS (AED)}

Inicialmente, realizou-se a análise exploratória dos dados (AED), com o objetivo de avaliar a consistência das respostas. Segundo Triola (2005), quando se trabalha com grande conjunto de dados, a AED é imprescindível para determinar a qualidade das análises decorrentes.

A AED permite ao pesquisador escolher os tipos de análises apropriados e dimensionar, também, a capacidade de extrapolação das conclusões do estudo. $\mathrm{O}$ que está intimamente ligado ao tipo de distribuição e à construção de métricas apropriadas. Nessa etapa, são comuns a eliminação de outiliers e a ponderação sobre a fidelidade dos construtos.

\subsection{ANÁLISE FATORIAL}

Após a eliminação de outliers e dos questionários inconsistentes, pelo procedimento de AED, realizou-se o procedimento de análise fatorial com as variáveis mais relevantes do estudo para obter número reduzido de fatores estratégicos. Segundo Hair (2005), em termos gerais, a análise fatorial aborda o problema de analisar estruturas das inter-relações entre grande número de variáveis, definindo um conjunto de dimensões latentes comuns. Essas dimensões são chamadas fatores. O procedimento utilizado para o cálculo dos fatores foi o dos componen- 
tes principais, por ser amplamente empregado na maioria dos estudos, sendo, também, o método mais adequado quando a preocupação principal é a previsão ou a obtenção de número mínimo de fatores necessários para explicar a máxima variância representada pelo conjunto original das variáveis.

Em geral, a análise fatorial deve ser realizada seguindo um conjunto de procedimentos estatísticos: a) determinação das correlações entre as variáveis selecionadas; b) extração de fatores significativos e necessários à representação dos dados; c) transformação dos fatores, por intermédio do procedimento de rotação, como forma de torná-los interpretáveis; e d) construção dos escores fatoriais.

A determinação das correlações permite excluir aquelas variáveis que não admitem relações com as demais. A segunda etapa consiste na ponderação e seleção do número de fatores necessários à explicação do conjunto de dados, bem como do método de cálculo para sua obtenção. O procedimento mais comum a esse respeito tem sido a utilização dos componentes principais para o cálculo dos fatores. Na terceira fase, rotação dos componentes principais, é comum a utilização do método Varimax, que visa minimizar o número de variáveis altamente relacionadas com cada um dos fatores, facilitando a interpretação dos resultados. O método Varimax tem sido muito bem-sucedido como abordagem analítica para a obtenção de rotação ortogonal de fatores (HAIR, 2005; MAROCO, 2003).

A quarta etapa consiste na obtenção dos escores fatoriais para cada unidade produtiva presente na amostra. O escore fatorial é resultante da multiplicação do valor padronizado da variável i pelo coeficiente do escore fatorial correspondente. $\mathrm{O}$ j-ésimo fator, $\mathrm{Fj}$, pode ser obtido pela equação I, adaptada de Manly (I986).

$$
F_{j}=\sum_{i=1}^{p} W_{j i} X_{I}=W_{j 1} X_{1}+W_{j 2} X_{2}+\ldots+W_{j p} X_{p}
$$

em que os Wji são os coeficientes dos escores fatorais e p é o número de variáveis.

\subsection{ANÁLISE DE AGRUPAMENTOS}

Após a redução dos fatores, foi realizada a análise de cluster, que tem como objetivo agrupar objetos ou indivíduos segundo suas características fundamentais, formando grupos ou conglomerados semelhantes. De acordo com Hair (2005), as organizações em cada conglomerado tendem a ser semelhantes entre si, porém diferentes das demais pertencentes aos outros conglomerados. Sendo este o mesmo propósito perseguido pela análise de agrupamentos estratégicos, torna-se, portanto, o procedimento multivariado mais adequado para os objetivos do trabalho. 
A análise de cluster envolve pelo menos dois momentos: a mensuração da similaridade ou associação entre as variáveis, determinando o número de grupos da amostra; e o esboço do perfil das variáveis, que determina a composição dos grupos.

O conceito implícito está associado à necessidade de se apropriar de algum procedimento para desvendar a relação de associação entre conjunto de componentes. Neste trabalho, o procedimento utilizado está pautado na distância entre os elementos. Assim, os agrupamentos resultam na homogeneidade derivada do menor somatório de distância entre os componentes pari passu com a maior heterogeneidade dos grupos.

Diferentes métodos podem ser usados para a operacionalização do cálculo das distâncias entre os objetos de estudo, os quais são classificados em hierárquicos e não-hierárquicos. Os procedimentos não-hierárquicos designam as observações aos grupos por intermédio de um processo em que o número de grupos deve ser previamente definido. Já os procedimentos hierárquicos consistem, basicamente, na formação de uma estrutura hierárquica, podendo seguir a via aglomerativa ou difusa.

Neste trabalho, utilizou-se o método hierárquico aglomerativo de Ward. O procedimento básico consiste em computar uma matriz de distância ou similaridade entre os indivíduos, a partir da qual se inicia um processo de sucessivas fusões destes, com base na proximidade ou similaridade entre eles. Essa matriz é simétrica $(\mathrm{dAB}=\mathrm{dAB})$, com zeros na diagonal principal, sendo obtida mediante vários métodos. Um dos mais comuns consiste no emprego do quadrado da distância euclidiana como medida de semelhança entre as observações, expressa matematicamente por:

$$
d_{(A, B)}=\left[\sum_{i=1}^{0}\left(X_{i(A)}-X_{i(B)}\right)^{2}\right]^{1 / 2}
$$

em que $d(A, B)$ é a medida de distância euclidiana da observação $\mathrm{A}$ a $\mathrm{B}$, sendo $\mathrm{i}$ o indexador das variáveis. Se a distância euclidiana for próxima a zero, significa que os objetos comparados são similares.

É válido ressaltar que não existe, nessa metodologia, nenhuma imposição ou restrição ao número de grupos considerados, dependendo do julgamento do pesquisador no que diz respeito ao foco do trabalho. Uma alternativa comumente utilizada pelos pesquisadores é a seleção dos grupos com base na análise do dendograma, estabelecendo-se um corte em dado valor da distância que melhor representa a distinção entre os grupos. Descrição detalhada dessa 
metodologia pode ser encontrada em Duran e Odell (I974), Manly (I986) e Johnson e Wichern (1998).

Nessa técnica de análise multivariada de dados, os conglomerados obtidos devem apresentar tanto homogeneidade interna (dentro de cada conglomerado) quanto grande heterogeneidade externa (entre conglomerados). Portanto, se a aglomeração for bem-sucedida, quando for representada em um gráfico, os objetos dentro dos conglomerados estarão muito próximos, e os conglomerados distintos estarão afastados.

Por fim, é comum nas análises em ciências sociais, a exemplo da administração e economia, dar nomes a cada agrupamento, de acordo com suas características. É comum realizar também uma análise descritiva comparativa, para a validação dos agrupamentos, tomando, como referência, variáveis selecionadas.

\section{RESULTADOS}

\subsection{ANÁLISE DOS FATORES ESTRATÉGICOS}

Visando agrupar as diversas variáveis de caracterização, estrutura e comportamento estratégico das unidades produtivas de lácteos, foi realizado o procedimento de análise fatorial.

O Quadro 2 apresenta as estatísticas descritivas das variáveis utilizadas na análise fatorial.

\section{QUADRO 2}

VARIÁVEIS UTILIZADAS NA CONSTRUÇÃO DOS FATORES ESTRATÉGICOS

\begin{tabular}{lcccc}
\hline & MÉDIA & DESVIO PADRÃO & ASSIMETRIA & CURTOSE \\
\hline $\begin{array}{l}\text { Volume recepção de leite em litros/ } \\
\text { dia }\end{array}$ & $9.192,05$ & $26.140,46$ & 7,12 & 63,45 \\
\hline Capacidade instalada em litros/dia & $20.680,76$ & $56.916,93$ & 7,01 & 59,74 \\
\hline Empregados & 19,14 & 37,58 & 4,54 & 24,20 \\
\hline $\begin{array}{l}\text { Patrimônio total em reais } \\
\text { Escala de experiência profissional do } \\
\text { dirigente }\end{array}$ & $1.346 .608,86$ & $5.073 .553,31$ & 8,01 & 75,73 \\
\hline
\end{tabular}




\section{QUADRO 2 (CONCLUSÃO)}

\section{VARIÁVEIS UTILIZADAS NA CONSTRUÇ ÃO}

DOS FATORES ESTRATÉGICOS

\begin{tabular}{lcccc}
\hline & MÉDIA & DESVIO PADRÃO & ASSIMETRIA & CURTOSE \\
\hline Escala de idade do dirigente & 3,89 & 1,00 & 0,17 & $(0,48)$ \\
\hline $\begin{array}{l}\text { Escala do grau de escolaridade do } \\
\text { dirigente }\end{array}$ & 2,84 & 0,92 & 0,48 & $(0,15)$ \\
\hline Número de produtos industrializados & 9,34 & 9,82 & 2,28 & 6,44 \\
\hline Número de marcas & 2,15 & 13,21 & 14,99 & 225,52 \\
\hline
\end{tabular}

Fonte: Resultados da pesquisa.

A análise fatorial resultou na extração de três fatores com raiz característica (eigenvalues) maiores que um e que respondem, em conjunto, por $73,80 \% \mathrm{da}$ variância total dos dados, conforme visualizado no Quadro 3.

As variáveis utilizadas apresentaram bom ajustamento representado pelo resultado do teste de Kaiser-Meyer-Olkin (KMO), com coeficiente de o,7I8 e consistência estatística representada pelo teste esferidade de Bartlett, significativo, a I\% de probabilidade.

\section{QUADRO 3}

CARACTERIZAÇÃO DOS PRINCIPAIS FATORES EXTRAÍDOS PELO MÉTODO DOS COMPONENTES PRINCIPAIS

\begin{tabular}{cccc}
\hline FATOR & RAIZ CARACTERISTICA & $\begin{array}{c}\text { VARIÂNCIA EXPLICADA } \\
\text { PELO FATOR (\%) }\end{array}$ & $\begin{array}{c}\text { VARIÂNCIA } \\
\text { ACUMULADA (\%) }\end{array}$ \\
\hline 1 & 3,64 & 43,97 & 43,97 \\
\hline 2 & 1,22 & 16,21 & 60,19 \\
3 & 1,04 & 13,61 & 73,80 \\
\hline
\end{tabular}

Fonte: Resultados da pesquisa.

Em função da análise das cargas fatoriais corresponderem aos coeficientes de correlação entre a variável i e o fator j, após rotação ortogonal pelo método Varimax, foi possível classificar três fatores e defini-los de acordo com a sua representação homogênea, conforme ilustrado no Quadro 4. Optou-se pela exposição, apenas, das variáveis com escore fatorial superior a 0,50 . 


\section{QUADRO 4}

\section{MATRIZ DE COMPONENTES APÓS ROTAÇÃO ORTOGONAL}

\begin{tabular}{lccc}
\hline & \multicolumn{3}{c}{ COMPONENTES } \\
\cline { 2 - 4 } & 1 & 2 & 3 \\
\hline Escala da experiência profissional do dirigente & & 0,833 & \\
\hline Escala de idade do dirigente & & & \\
\hline Volume recepção de leite em litros/dia & 0,957 & & 0,846 \\
\hline Capacidade instalada em litros/dia & 0,897 & \\
\hline Número de produtos industrializados & & & \\
\hline Número de marcas & & & \\
\hline Empregados & 0,936 & \\
\hline Patrimônio total em reais & 0,906 & \\
\hline
\end{tabular}

Fonte: Resultados da pesquisa.

- Fator 1 (tamanho): permite dimensionar o investimento humano e de capital da organização. Está diretamente relacionado às variáveis que expressam capacidade de recepção, volume de produção, patrimônio e recursos humanos.

- Fator 2 (experiência da gestão): assinala a experiência profissional, materializada na idade do dirigente e no número de anos em que este atua no setor de lácteos.

- Fator 3 (diversificação e diferenciação): caracteriza a organização em relação ao número de produtos e de marcas, representando a agregação de valor e a diversificação da cesta de produtos. A agregação de valor decorre diretamente das políticas de processamento e comercialização de produtos lácteos elaborados, em oposição às políticas de repasse ou negociação da produção de leite in natura. A diversificação diz respeito ao número de produtos processados pela organização.

\subsection{AGRUPAMENTOS ESTRATÉGICOS}

Para interpretação dos grupos formados, no intuito de discernir sobre a combinação dos fatores que levou à construção de cada agrupamento, foi realizada a 
análise dos escores fatoriais, classificados em baixo, médio e alto ${ }^{\mathrm{I}}$. O Quadro 5 resume a caracterização dos agrupamentos e apresenta o número de organizações em cada agrupamento.

\section{QUADRO 5}

ANÁLISE DE DESEMPENHO DOS GRUPOS

\begin{tabular}{|c|c|c|c|}
\hline $\begin{array}{l}\text { AGRUPAMENTOS } \\
\text { ESTRATÉGICOS }\end{array}$ & NÚMERO & FATORES & CLASSIFICAÇÃO \\
\hline $\begin{array}{l}\text { Cluster 1: } \\
\text { PP - Especializada }\end{array}$ & 147 & $\begin{array}{l}\text { F1 Tamanho } \\
\text { F2 Experiência da gestão } \\
\text { F3 Diversificação e diferenciação }\end{array}$ & $\begin{array}{l}\text { Baixo } \\
\text { Médio } \\
\text { Baixo }\end{array}$ \\
\hline $\begin{array}{l}\text { Cluster 2: } \\
\text { AP - Diversificada }\end{array}$ & 9 & $\begin{array}{l}\text { F1 Tamanho } \\
\text { F2 Experiência da gestão } \\
\text { F3 Diversificação e diferenciação }\end{array}$ & $\begin{array}{l}\text { Alto } \\
\text { Médio } \\
\text { Médio }\end{array}$ \\
\hline $\begin{array}{l}\text { Cluster 3: } \\
\text { MP - Diferenciada }\end{array}$ & 32 & $\begin{array}{l}\text { F1 Tamanho } \\
\text { F2 Experiência da gestão } \\
\text { F3 Diversificação e diferenciação }\end{array}$ & $\begin{array}{l}\text { Médio } \\
\text { Alto } \\
\text { Alto }\end{array}$ \\
\hline
\end{tabular}

Fonte: Resultados da pesquisa.

Por causa da combinação dos escores fatoriais, as organizações foram divididas em três grupos: pequeno porte (especializada), alto porte (diversificada) e médio porte (diferenciada).

A Figura I permite a visualização dos agrupamentos estratégicos em um plano de três dimensões, formado pelos fatores estratégicos.

I Os limites representam a média (O) e mais um (I/2) e menos um (I/2) desvio. 


\section{FigURA I}

AGRUPAMENTOS ESTRATÉGICOS NO SETOR DE LÁCTEOS

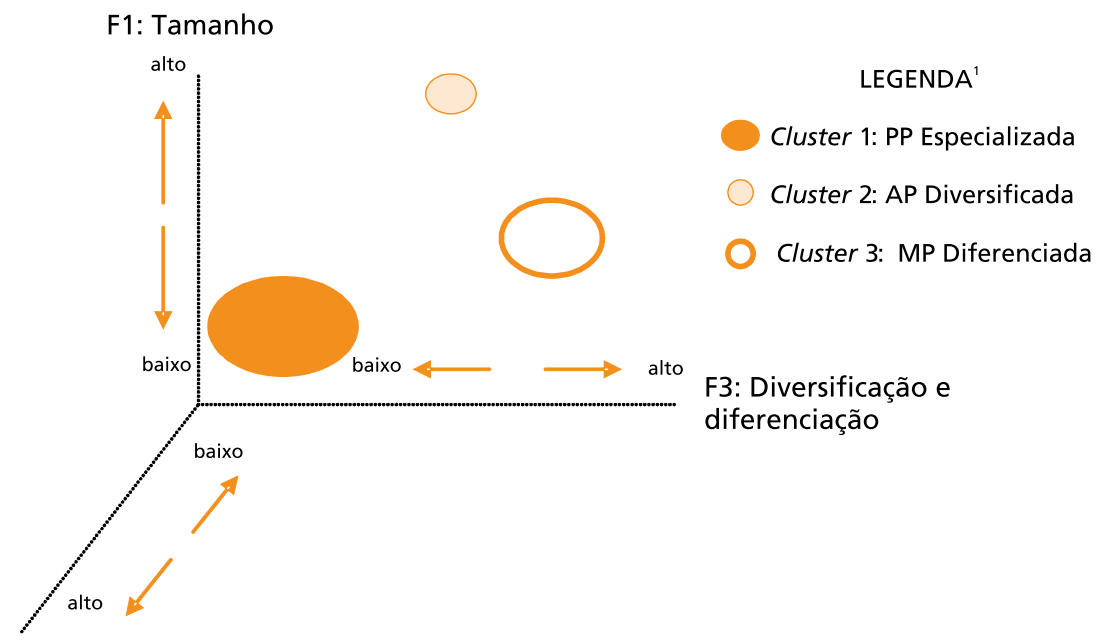

F2: Experiência e profissionalização

Fonte: Resultados da pesquisa.

As dimensões da Figura I representam os três fatores estratégicos utilizados na classificação dos grupos, a saber: FI - tamanho, F2 - experiência e profissionalização e $\mathrm{F}_{3}$ - diversificação e diferenciação. Para facilitar a visualização, os agrupamentos foram posicionados nas coordenadas dos fatores Fi e F3, que são mais relevantes na discriminação dos grupos, conforme apresentado no Quadro 5.

\subsection{AGRUPAMENTOS ESTRATÉGICOS}

\subsubsection{Cluster 1: organizações de pequeno porte e especializadas}

Essas organizações são caracterizadas por unidades de processamento de lácteos com baixo investimento humano e de capital, por limitada escala de processamento, pela incipiente agregação de valor ao produto e tímida variedade de produtos. Restringem-se à coleta e ao resfriamento do leite para a 
negociação com organizações maiores ou para o processamento de produtos de baixo valor agregado, como leite tipo $\mathrm{C}$, queijos brancos e manteiga. O incipiente investimento produtivo impossibilita o avanço em direção aos produtos de maior valor agregado e de maior elasticidade de renda, o que limita seu desempenho financeiro mesmo em períodos de aumento de poder aquisitivo e de consumo de lácteos.

\subsubsection{Cluster 2: organizações de alto porte diversificadas}

Esse agrupamento é constituído por unidades de processamento de lácteos que têm como característica fundamental o processamento em alta escala, associado ao elevado esforço de diversificação da produção.

A escala, associada à diversificação, permite que a organização atinja maior penetração de mercado e consolide marca forte lastreada ao portfólio diferenciado de produtos e aos esforços de pesquisas e desenvolvimento, mais comuns nesse agrupamento. Em conseqüência, espera-se que a empresa aumente sua capacidade produtiva, em decorrência da atração de maior número de consumidores.

A diferenciação, acompanhada de escala, permite também o acesso a vários mercados, não se restringindo à comercialização regional. Nesses mercados, o volume constitui em fator essencial na competitividade, na medida em que permite a elevação do poder de negociação com as grandes redes de aquisição. Pela maior penetração de mercados, as organizações de alto porte diversificadas estão competindo diretamente com grandes empresas nacionais e internacionais em um dos posicionamentos mais competitivos do mercado, isto é, a comercialização em nível do atacado.

\subsubsection{Cluster 3: organizações de médio porte diferenciadas}

Esse tipo de organização tem como característica fundamental o processamento em média escala, associado ao esforço elevado de agregação de valor ao produto. O agrupamento é caracterizado pela forte presença de empresas voltadas a "nichos específicos de mercado", embora produzam e comercializem em escala reduzida.

Essas organizações visam apropriar-se do que Porter (1986) definiu como estratégia competitiva no posicionamento mercadológico, ou seja, encontrar local defensivo no mercado e lograr dos benefícios deste.

Esse tipo de organização visa à consolidação de sua marca em mercados localizados via diferenciação de produtos, evitando o embate direto com grandes unidades produtivas, a exemplo de multinacionais e empresas nacionais de elevada expressão, muito comuns nessa indústria. 


\section{CONCLUSÕES}

A análise das unidades processadoras de lácteos possibilitou identificar a existência de fatores capazes de permitir a discriminação de grupos estratégicos na indústria de laticínios. Entre os fatores, tiveram destaque o tamanho da organização, a experiência da gestão, a diversificação de produtos e a capacidade de agregação de valor, via diferenciação.

Esses fatores reforçam a teoria científica, na medida em que abrem novas vertentes para as classificações das organizações, condicionadas, sobretudo, a elementos estratégicos, e permitem também operacionalizar análises estratificadas no setor.

A aplicação dos fatores na região de estudo provou ser eficaz na geração de agrupamentos coerentes com as proposições teóricas e práticas, em que se observou grande número de pequenas unidades, altamente especializadas, e reduzido número de organizações de grande porte, altamente diversificadas. Entre esses dois agrupamentos, observou-se a existência de organizações diferenciadas com apelo para nichos específicos de mercado, sem foco no processamento em grande escala.

Os agrupamentos, derivados da análise de cluster, têm a propriedade de criar informações preliminares imprescindíveis à realização de políticas públicas e privadas, que tenham como princípio a elevação do padrão de eficiência e competitividade das sociedades organizações que atuam no setor de processamento de lácteos.

\section{REFERÊNCIAS}

ATHANASSOPOUlOS, A. D. Goal programming and Data Envelopment Analysis (GoDEA) models for multi-level multi-unit organizations: an application to Greek local authorities. European Journal of Operational Research, v. 87, p. 535-550, I995.

ATHANASSOPOULOS, A. D.; BALLANTINE, J. Ratio and frontier analysis for assessing corporate performance: the case of grocery industry in the UK. Journal of the Operational Research Society, v. 46, p. 427-440, I995.

BARNEY, J. Firm resources and sustained competitive advantage. Journal of Management, v. I7, p. 77I-I992, I99I.

BARNEY, J.; HOSKISSON, R. Strategic groups: untested assertions and research proposals. Managerial and Decision Economics, v. II, n. 3, p. I87-198, I990.

BARTON, D. Principles. In: COBIA, D. Cooperatives in agriculture. New Jersey: Prentice Hall, I989. p. 2I-34. 
BOGNER, W.; MAHONEY, J. T.; THOMAS, H. Paradigm shift: the parallel origin, evolution, and function of strategic group with the resource-based theory of the firm. In: JOEL, A.; BAUM, C. (ed.). Disciplinary roots of strategic management research advances in strategic management. JAI Press, v. I5, p. 63-102, 1998.

BOGNER, W.; THOMAS, H.; McGEE, J. A longitudinal study of the competitive positions and entry paths of european firms in the US pharmaceutical industry. Strategic Management Journal, v. I7, p. 85-107, I996.

CAVES, R.; PORTER, M. From entry barriers to mobility barriers. Quarterly Journal of Economics, v. 9I, p. 24I-246, I977.

CHRISMAN, J. J.; HOFER, C. W.; BOULTON, W. R. Toward a system for classifying business strategies. Journal Academy of Management Review, v. I3, n. 3, p. 413-428, 1988.

COOK, M. L. The future of US Agricultural cooperatives: a neo-institutional approach. American Journal of Agricultural Economics, n. 77, p. II53-II59, I995.

COOK, M. L.; TONG, L. Definitional an classification issues in analyzing cooperative organizational forms. In: COOK, M. L. et al. Cooperatives: their importance in the future food and agricultural system. Ed. Michael Cook: Washington, I997. p. II3-II8. (Symposium sponsored by the National Council of Farmer Cooperatives and the Food and Agricultural Marketing Consortium). COOL, K.; SCHENDEL, D. Performance differences among strategic group members. Strategic Management Journal, v. 9, n. 3, p. 207-223, I988.

DAY, D.; LEWIN, A.; LI, H. Strategic leaders or strategic groups: a longitudinal data envelopment analysis of the US brewing industry. European Journal of Operational Research, v. 80, n. 3, p. 6I9638, I995.

DURAN, B. S.; ODELL, P. L. Cluster analysis - a survey. New York: Springer-Verlag, 1974- I37 p. DUYSTERS, G.; HAGEDOORN, J. Strategic groups and inter-firm networks in international high-tech industries. Journal of Management Studies, n. 32, p. 359-380, 1995.

EMBRAPA GADO DE LEITE. Banco de dados econômicos - 2006. Disponível em: <http://www. cnpgl.embrapa.br.>. Acesso em: 5 set. 2006.

FERREIRA, M. A. M. Eficiência técnica e de escala de cooperativas e sociedades de capital na indústria de laticínios do Brasil. 2005. 158 p. Tese (Doutorado em Economia Aplicada) - Universidade Federal de Viçosa, Viçosa, 2005.

FERREIRA, M. A. M.; BRAGA, M. J. Fatores internos associados à estratégia de diversificação nas cooperativas agropecuárias. Economia Aplicada, v. 7, n. 3, p. 607-632, 2003.

Diversificação e competitividade nas cooperativas agropecuárias. Revista de Administração Contemporânea (RAC), v. 8, n. 4, p. 33-55, 2004.

FERRIER, G. D.; PORTER, P. K. The productive efficiency of US milk processing co-operatives. Journal of Agricultural Economics, n. 42, p. I6I-I73, I99I.

FIEGENBAUM, A.; THOMAS, H. Strategic groups and performance: the U. S. insurance industry, 1970-84. Strategic Management Journal, v. II, n. 3, p. I97-215, I990.

. The concept of strategic groups as reference groups: an adaptive model and an empirical test. In: DAEMS, H.; THOMAS, H. (Ed.). Strategy groups, strategic moves and performance. Oxford: Pergamon, I994. 3I2 p.

FIEGENBAUM, A.; SUDHARSHAN, D.; THOMAS, H. The concept of stable strategic time periods in strategic group research. Managerial a Decision Economics, v. 8, n. 2, p. I39-I48, I993. 
- INVESTIGAÇÃO DE GRUPOS ESTRATÉGICOS NA INDÚSTRIA DE LATICÍNIOS... *

GORDON, M.; MILNE, G. Selecting the dimensions that define strategic groups: a novel market driven approach. Journal of Managerial Issues, v. II, n. 2, p. 213-233, I999.

HAIR, J. F. Análise multivariada de dados. Porto Alegre: Bookman, 2005. 593 p.

HATTEN, K.; SCHNEDEL, J. Heterogeneity within an industry. Journal of Industrial Economics, v. 26, p. 97-II3, I977.

HATTEN, K.; SCHNEDEL, D.; COOPER, A. A strategic model of the U. S. brewing industry: I952-I971. Academy of Management Journal, n. 21, p. 592-6Iо, I978.

HENDRIKSE, G. W. J.; VEERMAN, C. P. Marketing cooperatives as a system of attributes. In: NILSON, J.; VAN DIJK, G. Strategies and structures in the agrofood industries. Assen: Van Gorcum, I995. P. III-I3O.

HERBERT, T. T.; DERESKY, H. Generic strategies: an empirical investigation of typology and strategy content. Strategic Management Journal, n. 8, p. I35-I47, I987.

HERGERT, M. L. Causes and consequences of strategic grouping in U. S. manufacturing industries. International Studies of Management and Organization, n. I8, p. 26-49, I987.

JOHNSON, R.; WICHERN, D. W. Applied multivariate statistical analysis. 4. ed. New Jersey: Prentice Hall, I998.

KETCHEN, D.; SHOOK, C. The application of cluster analysis in strategic management research: an analysis and critique. Strategic Management Journal, v. I7, p. 44I-458, I993.

KUMAR, R.; THOMAS, H.; FIEGENBAUM, A. Strategic groupings as competitive benchmarks for formulating future competitive strategy: a modeling approach. Managerial Q Decision Economics, v. II, n. 2, p. 99-I09, I990.

KYRIAKOPOULOS, K. The market orientation of cooperative organizations: learning strategies and structures integrating firm and members. Assen: Van Gorcum, 2000. I68 p.

LANNES, J. Custo e diferenciação como condicionantes para a sobrevivência econômica das cooperativas de leite. In: BRAGA, M. J.; REIS, B. S. (Org.). Agronegócio cooperativo: reestruturação e estratégias. Viçosa: Universidade Federal de Viçosa, 2002. p. I4I-I56.

LAWLESS, M.; ANDERSON, P. Generational technological change: the effects of innovation and local rivalry on performance. Academy of Management Journal, p. 645-664, I996.

LEWIS, P.; THOMAS, H. The linkage between strategy, strategic groups, and performance in the UK retail grocery industry. Strategic Management Journal, v. II, n. 5, p. 385-397, I990.

Strategy, strategic groups and performance in industry. In: DAEMS, H.; THOMAS, H. (Ed.). Strategic groups, strategic moves and performance. Oxford: Pergamon, I994. p. 26I-278.

MAHONEY, J.; PANDIAN, R. The resource-based view within the conversation of strategic management. Strategic Management Journal, v. I3, p. 363-380, I992.

MANLY, B. F. J. Multivariate statistical methods - a primer. New York: Chapman and Hall, I986. I59 p.

MAROCO, J. Análise estatística. Lisboa: Sílabo, 2003. 508 p.

MATIAS, A. Aplicação da metodologia dos grupos estratégicos: o caso da indústria hoteleira no Algarve. Revista Portuguesa de Gestão, p. 3I-46, I992.

MELO, J. L. Dinâmica concorrencial da indústria de laticínios no Brasil na década de 90. 2003. I58 f. Tese (Doutorado em Economia Aplicada) - Universidade Federal de Viçosa, Viçosa, 2003.

MURRAY, G. C. Towards an agricultural cooperative classification. Journal of Agricultural Economics, v. 34, n. 2, p. I5I-I6I, I983. 
NATH, D.; GRUCA, T. Convergence across alternative methods for forming strategic groups. Strategic Management Journal, v. I8, n. 9, p. 745-760, I997.

NILSSON, J. Organizational principles for cooperative firms. Scandinavian Journal of Management, n. I7, p. 329-356, 1999.

PENROSE, E. G. The theory of the growth of the firm. 2. ed. Oxford: Basil BlackWell, I959. 272 p.

PETERAF, M.; SHANLEY, M. Getting to know you: a theory of strategic group identity. Strategic Management Journal, v. I8, p. I65-186, I997.

PORTER, M. E. Estratégia competitiva: técnicas para a análise de indústrias e da concorrência. 7 . ed. Rio de Janeiro: Campus, I986.362 p.

REGER, R.; HUFF, A. Strategic groups: a cognitive perspective. Strategic Management Journal, n. I4, p. IO3-I24, I993.

SCHEFCZYK, M. Operational performance of airlines: an extension of traditional measurement paradigms. Strategic Management Journal, n. I4, p. 30I-3I7, I993.

SCHENDEL, D. E.; HOFER, C. W. Strategic management: a new view of business policy and planning. Boston, MA: Little, Brown, I979.

THOMAS, H.; CARROLL, C. Theoretical and empirical links between strategic groups, cognitive communities and networks of interacting firms. In: DAEMS, H.; THOMAS, H. (Ed.). Strategic groups, strategic moves and performance. Oxford: Pergamon, I994.

TRIOLA, M. F. Introdução à estatística. Rio de Janeiro: LTC, 2005. 656 p.

VAN BEKKUM, O. F. Cooperatives models and farm policy reform. Netherlands: Van Gorcum, $200 \mathrm{I}$. 23I p.

VAN DIJK, G. The changing theorical perspective of cooperatives and markets. In: EGERSTROM, L.; BOS, P.; VAN DIJK, G. Seizing control: the international market power of cooperatives. Rochester Minnesota: Lone Oak Press, I996. p. I70-I86.

WRIGHT, P. et al. Strategic profiles and performance: an empirical test of select key propositions. Journal of the Academy of Marketing Science, v. 3, n. I9, p. 245-254, I991.

\section{TRAMITAÇ ÃO}

Recebido em 1요 $/ 8 / 2007$

Aprovado em 3/10/2007 